\title{
EL CARIOTIPO DE LAPAGERIA ROSEA RUIZ ET PAV. (LILIALES: PHILESIACEAE)
}

\section{THE KARYOTYPE OF LAPAGERIA ROSEA RUIZ ET PAV. (LILIALES: PHILESIACEAE)}

\author{
Pedro Jara-Seguel \& Cristina A. Zúñiga \\ Departamento de Ciencias Biológicas y Químicas, Facultad de Ciencias, Universidad Católica de Temuco, Casilla 15- \\ D, Temuco-Chile.E-mail: pjara@uct.cl
}

\begin{abstract}
Lapageria rosea Ruiz et Pav. showed an asymmetric and bimodal karyotype $2 n=2 x=30$, with three metacentric, six submetacentric and six subtelocentric chromosome pairs. One B chromosome pair was also observed. The total chromosome length $(2 \mathrm{n})$ was $131.3+0.22 \mu \mathrm{m}$.
\end{abstract}

Con el objeto de ampliar la información citogenética existente para Philesiaceae, este trabajo provee una descripción cuantitativa de la morfología cromosómica de Lapageria rosea. Watson \& Dallwitz (1992), en una revisión de caracteres diagnósticos de angiospermas, señalan para Philesiaceae los números cromosómicos básicos $\mathrm{x}=15 \mathrm{y} 19$. Previamente a estos datos, se describió para Lapageria rosea Ruiz et Pav, un cariotipo $2 \mathrm{n}=2 \mathrm{x}$ $=30(\mathrm{x}=15)($ Titov de Tschicshow 1954), por lo que $\mathrm{x}=19$ podría corresponder al número básico de Philesia magellanica J. F. Gmel. Sin embargo, estos antecedentes basados en números cromosómicos no son suficientes para relacionar a Lapageria y Philesia, por lo que es necesario recopilar datos acerca de morfología y tamaño cromosómico. Los datos citogenéticos son importantes, también, para evaluar el grado de diversidad genómica dentro del orden Liliales (Bennett \& Leitch 1997), así como para futuros análisis genético-poblacionales relacionados con endogamia, tal como lo sugiere Henríquez (2004) al estudiar el efecto de la fragmentación del hábitat sobre la calidad de las semillas de L. rosea. Para Liliales chilenos, solamente el género Alstroemeria (Alstroemeriaceae) cuenta con una amplia base de datos citogenéticos que incluyen morfología cromosómica, contenido de ADN y patrones de bandeo C (Buitendijk \& Ramanna 1996, Buitendijk et al. 1997, Sanso \& Hunziker 1998, Sanso 2002, Jara-Seguel et al. 2004). Estos datos citogenéticos han sido útiles para evaluar relaciones intra e interespecíficas en este género, las cuales son consistentes con filogenias morfológicas y moleculares documentadas por Aagesen \& Sanso (2003).

Frutos de una población silvestre de L. rosea (variedad roja, ID: PJ0204) fueron colectados en el Campus Norte de la Universidad Católica de Temuco $\left(38^{\circ} 41^{\prime} \mathrm{S} ; 7^{\circ} 33^{\prime} \mathrm{W}\right)$ y algunos ejemplares fueron depositados en el Herbario (H-UCT) del Departamento de Ciencias Biológicas y Químicas de la misma universidad. Raíces de semillas germinadas fueron tratadas con colchicina $0,05 \%(\mathrm{p} / \mathrm{v})$ por $3 \mathrm{~h}$, fijadas en etanol-ácido acético $(3: 1 \mathrm{v} / \mathrm{v})$ a $4^{\circ} \mathrm{C}$ por $24 \mathrm{~h}$ y coloreadas mediante la reacción de Feulgen. Los cromosomas se obtuvieron por aplastado de meris-temas radiculares. A partir de fotomicrografías de diez metafases (obtenidas de semillas de diez plantas) se determinó la longitud de brazos cortos y largos. Las mediciones fueron expresadas como porcentaje de la longitud total del conjunto haploide. Con estos valores se determinó la morfología cromosómica siguiendo a Levan et al. (1964), se estimó la longitud cromosómica total (en $\mu \mathrm{m}$ ) y el nivel de asimetría cariotípica de acuerdo a Stebbins (1971). Los cromosomas se ordenaron considerando longitud relativa (LR) y posición del centrómero. 
Lapageria rosea presenta un cariotipo $2 \mathrm{n}=2 \mathrm{x}$ $=30$, con tres pares de cromosomas metacéntricos, seis submetacéntricos y seis subtelocéntricos, además de un par de cromosomas B. (Fig. 1, Tabla I). No se observó la presencia de satélites. El par 1, submetacéntrico grande, duplica en longitud relativa a los restantes pares cromosómicos del complemento ( $\mathrm{LR}=18,9 \%)$, por lo que este cariotipo podría clasificarse como bimodal. El nivel de asimetría del cariotipo es 2C, de acuerdo a las categorías de Stebbins (1971). La longitud cromosómica total (2n) es de 131,3 $+0,22 \mu \mathrm{m}$, con un tamaño cromosómico promedio de $4,37+2,64 \mu \mathrm{m}$. El tamaño promedio de los cromosomas B es de 1,13 $+0,04 \mu \mathrm{m}$ y no fue posible identificar sus centrómeros.

El número cromosómico y la morfología del cariotipo de $L$. rosea descritos en este trabajo confirman los datos de Titov de Tschicshow (1954). Ese estudio, mediante cortes histológicos de botones florales, describió quince pares de cromosomas acrocéntricos y en cuyo idiograma es posible determinar morfologías cromosómicas metacéntricas, submetacéntricas, subtelocéntricas y telocéntricas de acuerdo a las categorías propuestas por Levan et al. (1964).

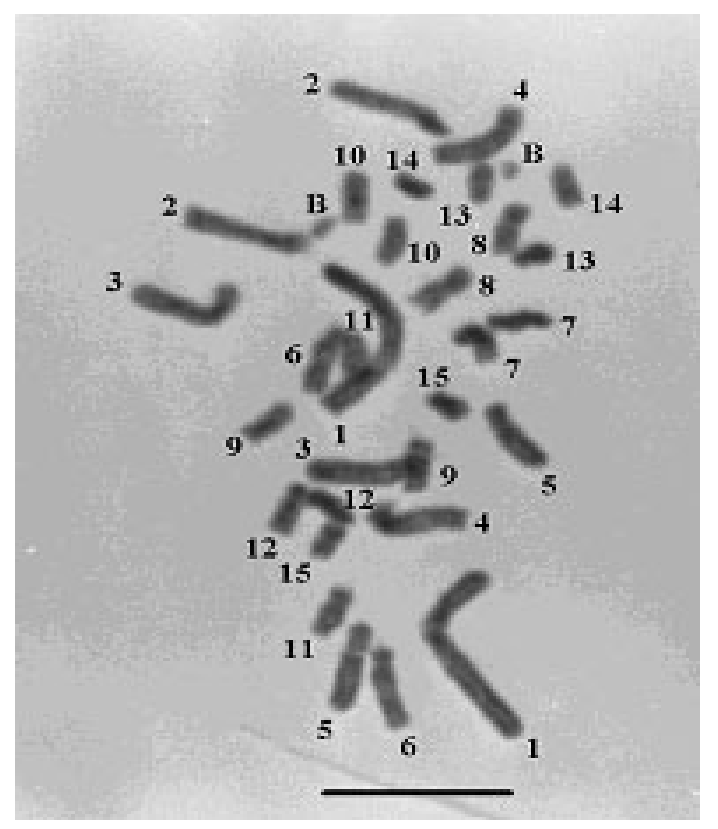

FIgURA 1. Cromosomas mitóticos de Lapageria rosea, $2 \mathrm{n}$ $=2 \mathrm{x}=30+2 \mathrm{~B}$. Barra $=10 \mu \mathrm{m}$.

TABLA I. Longitud relativa (LR: \%), longitud total (LT: $\mu \mathrm{m}$ ), índice centromérico (IC) y morfología (M) de cada par cromosómico de Lapageria rosea $(\mathrm{n}=10$ metafases). d.e. = desviación estándar.

\begin{tabular}{|c|c|c|c|c|c|c|c|c|c|c|c|c|c|c|c|}
\hline \multicolumn{16}{|c|}{ Par cromosómico } \\
\hline & 1 & 2 & 3 & 4 & 5 & 6 & 7 & 8 & 9 & 10 & 11 & 12 & 13 & 14 & 15 \\
\hline LR & 18,9 & 10,1 & 9,5 & 8,9 & 8,2 & 7,0 & 5,2 & 5,1 & 5,1 & 4,4 & 4,4 & 4,4 & 3,2 & 3,2 & 2,5 \\
\hline LT & 12,46 & 6,65 & 6,23 & 5,82 & 5,40 & 4,57 & 3,34 & 3,32 & 3,32 & 2,91 & 2,91 & 2,91 & 2,08 & 2,08 & 1,66 \\
\hline+ d.e. & 0,05 & 0,02 & 0,04 & 0,05 & 0,04 & 0,05 & 0,02 & 0,02 & 0,03 & 0,01 & 0,04 & 0,03 & 0,05 & 0,03 & 0,05 \\
\hline IC & 0,36 & 0,19 & 0,20 & 0,21 & 0,30 & 0,18 & 0,22 & 0,50 & 0,25 & 0,28 & 0,28 & 0,28 & 0,40 & 0,37 & 0,50 \\
\hline M & $\mathrm{sm}$ & st & st & st & $\mathrm{sm}$ & st & st & $\mathrm{m}$ & st & $\mathrm{sm}$ & $\mathrm{sm}$ & $\mathrm{sm}$ & $\mathrm{m}$ & $\mathrm{sm}$ & $\mathrm{m}$ \\
\hline
\end{tabular}

La longitud cromosómica total obtenida en este trabajo para $L$. rosea $(131,3 \mu \mathrm{m})$ se relaciona con un tamaño genómico $2 \mathrm{C}=13,56 \mathrm{pg}(4 \mathrm{C}=27,12$ $+1,95$ pg) (Hanson et al. 2003). Esta relación es distinta a la encontrada por Buitendijk et al. (1997) para especies de Alstroemeria $(2 \mathrm{n}=2 \mathrm{x}=16)$, cuyos rangos $2 \mathrm{C}$ están considerados entre los más altos dentro del reino Plantae, con valores que varían desde 36,5 hasta 78,9 pg y que se relacionan con longitudes cromosómicas totales $(2 \mathrm{n})$ dentro de un 
rango de 116,0 a 180,0 $\mu \mathrm{m}$ (Buitendijk \& Ramanna 1996).

La presencia de cromosomas B es un carácter variable en monocotiledóneas, siendo frecuentes en el cariotipo de varias especies de Liliales (Jones \& Rees 1982). En especies chilenas de este orden, cromosomas B han sido descritos en Alstroemeria angustifolia Herb. ssp. angustifolia (Buitendijk \& Ramanna 1996), Alstroemeria hoockerii Lodd. ssp. recumbens (Sanso 2002) y Lapageria rosea (Hanson et al. $2003 \mathrm{y}$ en el presente trabajo). La ventaja adaptativa de la presencia de cromosomas B ha sido discutida por Stebbins (1971), quien los relaciona con caracteres como el vigor y la fertilidad de las plantas. Es probable que, en L. rosea, la presencia de cromosomas B también esté relacionada con vigor y fertilidad, lo cual podría responder, al menos parcialmente, a las interrogantes planteadas por Henríquez (2004).

Finalmente, señalamos la importancia de realizar estudios cariológicos en Philesia magellanica, la segunda especie de Philesiaceae que habita en el sur de Chile (Watson \& Dallwitz 1992). Esto permitiría establecer relaciones citogenéticas dentro de la familia, de manera que puedan ser contrastadas con las actuales hipótesis filogenéticas basadas en la secuenciación de genes nucleares (Martin \& Dowd 1991) y de cloroplastos (Vinnersten \& Bremer 2001).

\section{AGRADECIMIENTOS}

AAlicia Marticorena, por facilitar bibliografía referente a estudios citológicos en $L$. rosea. A Enrique Hauenstein, por la lectura del manuscrito. Financiado por DIPUCT.

\section{BIBLIOGRAFIA}

Aagesen, L. \& M. SAnso. 2003. The phylogeny of the Alstroemeriaceae, based on morphology, rps 16 intron, and $r b c L$ sequence data. Systematic Botany 28(1): 47-69.
BenNett, M. \& I. Leitch. 1997. Nuclear DNA amounts in Angiosperms - 583 new estimates. Annals of Botany 80: 169-196.

BuitendiJK, J. \& M. Ramanna. 1996. Giemsa C-banded karyotypes of eigth species of Alstroemeria L. and some of their hybrids. Annals of Botany 78: $449-457$.

BuitendiJK, J., E. Boon \& M. Ramanna. 1997. Nuclear DNA content in twelve species of Alstroemeria L. and some of their hybrids. Annals of Botany 79: 343 - 353 .

Hanson, L., R. Brown, A. Boyd, M. Johnson \& M. BENNETT. 2003. First nuclear DNA C-values for 28 angiosperm genera. Annals of Botany 91: 3138.

Henriquez, C. 2004. Efecto de la fragmentación del hábitat sobre la calidad de las semillas en Lapageria rosea. Revista Chilena de Historia Natural 77: 177-184.

Jara-Seguel, P., C. Palma-Rojas \& E. Von Brand. 2004. Karyotype and C-bands in the annual Inca Lily Alstroemeria graminea Phil. Belgian Journal of Botany (en prensa)

Jones, R. \& H. Rees. 1982. B chromosomes. Academic Press London. 266 pp.

Levan, A., K. Fredga \& A. SANDBerg. 1964. Nomenclature for centromeric position on chromosomes. Hereditas 52: 201-220.

Martin, P. \& J. Dowd. 1991. A comparison of $18 \mathrm{~S}$ ribosomal RNA and rubisco large subunit sequences for studying angiosperm phylogeny. Journal of Molecular Evolution 33: 274-282.

SAnso, M. \& J. HunziKER. 1998. Karyological studies in Alstroemeria and Bomarea (Alstroemeriaceae). Hereditas 129: 67-74.

SAnso, M. 2002. Chromosome Studies in Andean taxa of Alstroemeria (Alstroemeriaceae). Botanical Journal of the Linnean Society 138: 451-459.

StebBins, G. 1971. Chromosomal Evolution in Higher Plants. Edward Arnold Publishing London. 215 pp.

Titov DE Tschicshow, N. 1954. Estudios citológicos en Lapageria rosea Ruiz et Pavon. Boletín de la Sociedad de Biología de Concepción 29: 3-6.

VinNERSTEN, A. \& K. BREMER. 2001. Age and biogeography of major clades in Liliales. American Journal of Botany 88: 1695-1703.

Watson, L. \& J. Dallwitz. 1992. The families of flowering plants, illustrations, identification, and information retrieval. http://www.biodiversity.uno.edu/delta/ Viewed: December 14, 2000 . 